\title{
Atrial Flutter With Exercise-Induced 1:1 Atrioventricular Conduction
}

Ryan Burkhart, DO; Gregg Gerasimon, MD

From the Division of Cardiology at the San Antonio Military Medical Center in Fort Sam in Houston, Texas. Financial Disclosures: None reported.

Support: None reported.

Disclaimer: The views expressed herein are those of the authors and do not necessarily reflect the official policy or position of the Department of the Army, the Department of Defense, or the US Government.

Address correspondence to Ryan Burkhart, DO, Division of Cardiology, San Antonio Military Medical Center, 3551 Roger Brooke Dr, JBSA Fort Sam, Houston, TX 782344504.

Email: ryan.v.burkhart.mil@ mail.mil Submitted May 4, 2017; revision received August 11, 2017; accepted September 13, 2017
Typical atrial flutter is a common atrial tachyarrhythmia. Symptoms are typically related to the rate of ventricular response. Although atrial flutter with 1:1 atrioventricular conduction is rare, it is important to recognize because it may precipitate rapid hemodynamic compromise. The authors present a case of exercise-induced 1:1 atrioventricular conduction in a patient with typical atrial flutter.

J Am Osteopath Assoc. 2018;118(5):337-340

doi:10.7556/jaoa.2018.065

Keywords: arrhythmia, atrial flutter, atrioventricular conduction

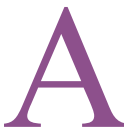
trial flutter is a commonly encountered arrhythmia in clinical practice, with an estimated incidence of 88 per 100,000 person-years. ${ }^{1}$ The incidence of typical atrial flutter increases with age, comorbidities, and any disease process that results in secondary atrial dilation. ${ }^{1}$ Risk factors for developing atrial flutter include hypertension, heart failure, valvular disease, acute and chronic lung disease, alcohol use, and metabolic disturbances. ${ }^{1}$ Atrial flutter typically results in an atrial rate of 250 beats/min to 350 beats $/ \mathrm{min}^{2}$ The ventricular rate usually occurs in multiples of the underlying atrial fibrillation rate (eg, 2:1, 3:1, 4:1), as the physiologic properties of the atrioventricular node limit the number of impulses that can reach the His-Purkinje system. Atrial flutter often causes a ventricular rate of 150 beats/min in the absence of atrioventricular nodal blocking agents or atrioventricular nodal disease. Symptoms are often related to the rate of ventricular response and may include palpitations, dyspnea, chest pain, presyncope, or syncope. Atrial flutter with 1:1 atrioventricular conduction is a rare entity and has classically been described in the setting of class $1 \mathrm{~A}$ or $1 \mathrm{C}$ antiarrhythmic drug therapy. ${ }^{3}$ This report describes a case of atrial flutter with exercise-induced $1: 1$ atrioventricular conduction during an exercise stress test.

\section{Report of Case}

A 71-year-old man with a history of coronary artery disease and a 1-vessel coronary artery bypass graft 14 years earlier was admitted to the hospital with the diagnosis of typical atrial flutter with a rapid ventricular response (ventricular rate, 130 beats $/ \mathrm{min}$ ). His symptoms included sudden-onset dyspnea, lightheadedness, and chest tightness during peak exertion during the past year, which would resolve shortly after cessation of exercise. He denied noticing any palpitations and also denied a history of syncope. His comorbidities included type 2 diabetes mellitus, obstructive sleep apnea, hypertension, and hyperlipidemia. His current medications included metoprolol succinate, $25 \mathrm{mg} / \mathrm{d}$; lisinopril, $20 \mathrm{mg} / \mathrm{d}$; aspirin, $81 \mathrm{mg} / \mathrm{d}$; atorvastatin, $40 \mathrm{mg} / \mathrm{d}$; and metformin, $750 \mathrm{mg} / \mathrm{d}$. 


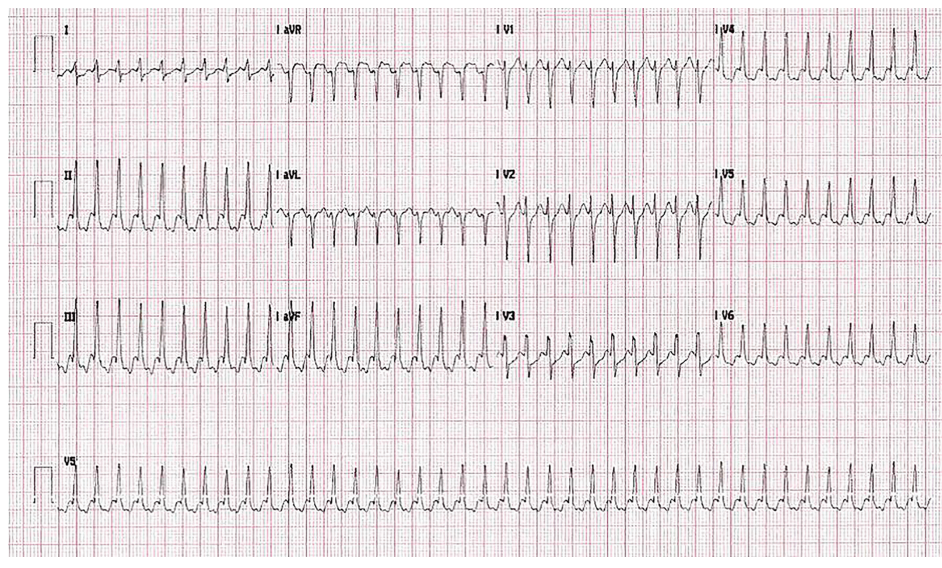

Figure 1.

A 1:1 artioventricular conduction with a ventricular rate of 240 beats $/ \mathrm{min}$ developed in a 71-year-old man 1 minute and 30 seconds into the treadmill test portion of an exercise stress test.

The patient underwent a myocardial perfusion exercise stress test the day after admission. One minute and 30 seconds into the treadmill test, a 1:1 atrioventricular conduction of the atrial flutter with a ventricular rate of 240 beats/min developed (Figure 1). The treadmill test was urgently stopped, and after 2 minutes of recovery, symptoms resolved and 2:1 atrioventricular conduction resumed. The cardiology department was then consulted for management. A transthoracic echocardiogram showed a mildly reduced ejection fraction of $40 \%$ to $45 \%$. Because of the reduced ejection fraction and the history of known coronary artery disease, a coronary angiogram recommended. Ventricular rate control (Figure 2) was achieved by titrating metoprolol tartrate to $100 \mathrm{mg}$ twice daily, and the patient was given $20 \mathrm{~m} / \mathrm{g}$ of rivaroxaban daily for stroke prevention. The patient was discharged the next day at his own request.

The patient presented to the cardiology department 5 days later for a coronary angiogram, which showed a patient bypass graft and severe native coronary artery disease. In the absence of any new obstructive lesion, the reduced ejection fraction was thought to be due to tachycardia-induced cardiomyopathy, given the persistent atrial flutter and elevated ventricular rates. He was referred to the cardiac electrophysiology department for a typical atrial flutter ablation, which he underwent 2 weeks after initial presentation. The atrial flutter ablation was successful, but severe underlying sinus node dysfunction was unmasked immediately after the ablation. Sinus node dysfunction did not recover, and the patient underwent permanent pacemaker implantation 2 days after the ablation. He was discharged the next day.

\section{Discussion}

Typical atrial flutter is a macroreentrant rhythm in the right atrium, with an atrial rate between 250 and 350 beats/min. ${ }^{2}$ Its circuit involves the cavotricuspid isthmus, with the arrhythmia often being termed cavotricuspid isthmus-dependent atrial flutter. This condition can degenerate into atrial fibrillation and can revert to sinus rhythm within hours or days. ${ }^{4}$ Management of atrial flutter is similar to that of atrial fibrillation in terms of symptom management, ventricular rate control, stroke prevention, and consideration for restoration of sinus rhythm. Ventricular rate control is often more difficult to achieve with atrial flutter than with atrial fibrillation. ${ }^{5}$

Atrial flutter with 1:1 atrioventricular conduction has commonly been described in the setting of class $1 \mathrm{~A}$ or $1 \mathrm{C}$ antiarrhythmic drug therapy, as these medications are known to slow the atrial flutter rate and, thereby, increase the potential for 1:1 conduction through the atrioventricular node. ${ }^{3}$ Because of the risk of 1:1 atrioventricular conduction during atrial flutter, class $1 \mathrm{~A}$ or 1C antiarrhythmic medications should not be prescribed to patients with a history of atrial flutter or atrial fibrillation without the addition of an atrioventricular nodal blocking therapy, such as beta blockers or nondihydropyridine calcium channel blockers. One-to-one atrioventricular conduction of atrial flutter may also occur in the presence of catecholamine excess, thyrotoxicosis, ventricular preexcitation, or marked right atrial enlargement. Atrial flutter is a potential cause of presyncope and syncope and can precipitate hemodynamic compromise quickly if it sustains, ${ }^{6}$ especially in patients with a reduced ejection fraction. ${ }^{7}$ During exercise, there is an increase in atrial flutter cycle length, a withdrawal 
of vagal tone, and an increase in sympathetic activation that facilitate atrioventricular nodal conduction. ${ }^{8}$ One-to-one atrioventricular conduction is more likely to occur when atrial rates are less than 250 beats $/ \mathrm{min}$. ${ }^{9}$

In the current case, the patient transitioned to $1: 1$ atrioventricular conduction with a ventricular rate of 240 beats/min during exercise. In instances in which 1:1 atrioventricular conduction develops, initial management should focus on gaining rate or rhythm control to maintain hemodynamic stability and reduce symptoms. Synchronized direct-current cardioversion must be considered for immediate rhythm control in a hemodynamically unstable patient. Long-term options for ventricular rate control include beta blockers, nondihydropyridine calcium channel blockers, digoxin, and amiodarone hydrochloride. Esmolol is often the preferred beta blocker because of its rapid onset and offset and because it is easily titratable. Nondihydropyridine calcium channel blockers, such as diltiazem, can be used for ventricular rate control as well; however, these medications must be used with caution if the ejection fraction is reduced, as they may precipitate heart failure. Digoxin works by enhancing parasympathetic tone and, thus, would be only minimally effective for ventricular rate control in the setting of exercise when sympathetic tone predominates. Amiodarone has a beta-blocking effect and can be used for ventricular rate control acutely; however, it has a long-term adverse effect profile and can precipitate restoration of sinus rhythm. If restoration of sinus rhythm is being pursued, stroke risk must be considered, and the left atrial appendage should be cleared from potential thrombus before restoring sinus rhythm. In the current case, because the 1:1 atrioventricular conduction was short lived, the patient did not require acute intervention aside from stopping the stress test, and he remained clinically stable.

An important consideration when attempting to restore sinus rhythm is that severe sinus node dysfunction may be unmasked after the termination of the atrial flutter. As a result, options for transvenous or transcutaneous pacing should be ready for use if a viable native rhythm does not occur after atrial flutter termination. In patients

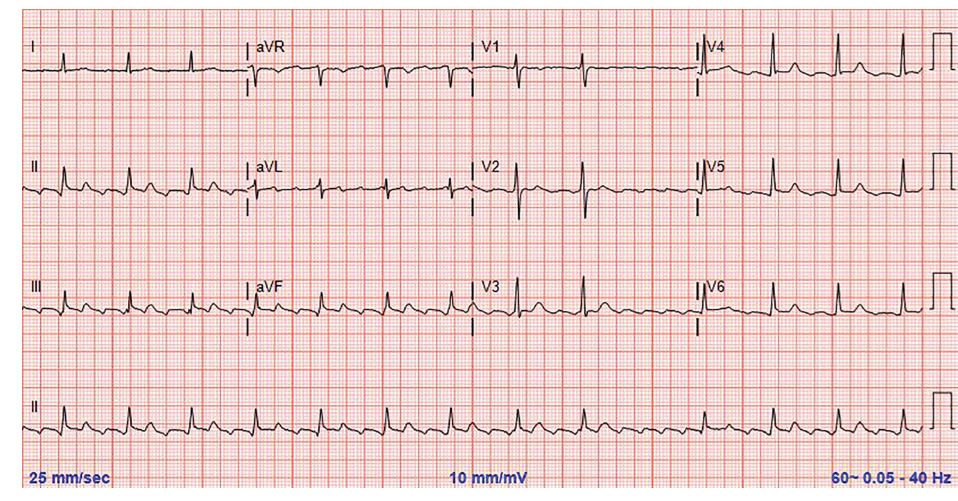

Figure 2.

Ventricular rate control was achieved in a 74-year-old man after developing atrial flutter with exercise-induced 1:1 atrioventricular conduction 1 week earlier.

with an atrial flutter, the ventricular rate is often more difficult to control than in patients with atrial fibrillation, and, thus, a rhythm control strategy is often pursued rather than a rate control strategy in patients with atrial flutter. ${ }^{5}$ The high success rate of atrial flutter ablation combined with its low complication rate make catheter ablation a popular management option.

There is a limited role for exercise stress testing in patients who present with atrial flutter. Indications for exercise stress testing in patients with atrial flutter are not specifically addressed in current guidelines. ${ }^{10}$ One potential role for exercise stress testing is to objectively determine whether a patient has adequate ventricular rate control during activity when a long-term rate control strategy is pursued. Type $1 \mathrm{C}$ antiarrhythmic agents have a greater propensity to bind to channels in their active state and display use-dependent pharmacokinetics. Exercise stress testing can screen for raterelated widening of the QRS complex and exercise-induced arrhythmia, though this testing is not routinely performed. ${ }^{11}$ For the evaluation of ischemia, standard indications and contraindications for stress testing would apply.

The limitations of exercise stress testing for patients with active atrial flutter should be understood. Flutter waves may limit the interpretability of the ST segment assessment and, in such cases, the addition of an imaging modality would be required. The 
patient's ventricular rate should be controlled before the stress test, as baseline rapid ventricular response is a contraindication. The difficulty of achieving adequate ventricular rate control and the propensity to developing rapid ventricular response, particularly with activity, limits the utility of exercise stress testing for evaluation of ischemia in patients with active atrial flutter. In these cases, it is important to be prepared for management of 1:1 atrioventricular conduction, should it occur.

\section{Conclusion}

Typical cavotricuspid isthmus-dependent atrial flutter is a common atrial tachyarrhythmia. ${ }^{1}$ Atrial flutter with 1:1 atrioventricular conduction can be precipitated by an exercise stress test. Therefore, there is a limited role for exercise stress tests in patients who present with atrial flutter. It is crucial to be cognizant of the clinical scenarios that can precipitate 1:1 atrioventricular conduction of atrial flutter, as well as the management strategies.

\section{References}

1. Granada J, Uribe W, Chyou PH, et al. Incidence and predictors of atrial flutter in the general population. J Am Coll Cardiol. 2000;36 (7):2242-2246
2. Wellens HJ. Contemporary management of atrial flutter. Circulation. 2002;106(6):649-652.

3. Aliot E, Capucci A, Crijns HJ, Goette A, Tamargo J. Twenty-five years in the making: flecainide is safe and effective for the management of atrial fibrillation. Europace. 2011;13(2):161-173. doi:10.1093/europace/euq382

4. Lee KW, Yang Y, Scheinman MM. Atrial flutter: a review of its history, mechanisms, clinical features, and current therapy. Curr Probl Cardiol. 2005;30(3):121-167. doi:10.1016/j.cpcardiol.2004.07.001

5. Cosío FG. Atrial flutter, typical and atypical: a review. Arrhythm Electrophysiol Rev. 2017;6(2):55-62. doi:10.15420/aer.2017:5:2

6. Kawabata M, Hirao K, Higuchi K, et al. Clinical and electrophysiological characteristics of patients having atrial flutter with 1:1 atrioventricular conduction. Europace. 2008;10(3):284-288. doi:10.1093/europace/eun012

7. Turitto G, Akhrass P, Leonardi M, Saponieri C, Sette A, El-Sherif N Atrial flutter with spontaneous 1:1 atrioventricular conduction in adults: an uncommon but frequently missed cause for syncope/presyncope. Pacing Clin Electrophysiol. 2009;32(1):82-90. doi:10.1111 jj.1540-8159.2009.02180.x

8. van den Berg MP, Crijns HJ, Szabó BM, Brouwer J, Lie KI. Effect of exercise on cycle length in atrial flutter. Br Heart J. 1995;73(3):263-264

9. Gooch AS, Sumathisena DR. The influence of exercise on atrial flutter. J Electrocardiol. 1975;8(1):39-48.

10. January CT, Wann LS, Alpert JS, et al. 2014 AHA/ACC/HRS guideline for the management of patients with atrial fibrillation: a report of the American College of Cardiology/American Heart Association Task Force on Practice Guidelines and the Heart Rhythm Society. J Am Coll Cardiol. 2014;64(21):e1-e76. doi:10.1016/j.jacc.2014.03.022

11. Vallurupalli S, Pothineni NVK, Deshmukh A, Paydak H. Utility of routine exercise testing to detect rate-related qrs widening in patients without structural heart disease on class Ic antiarrhythmic agents (flecainide and propafenone). Am J Cardiol. 2015;116(5):730-732. doi:10.1016/j.amjcard.2015.05.039

(c) 2018 American Osteopathic Association 